\title{
Vector valued Orlicz-Lorentz sequence spaces and their operator ideals
}

\author{
S. A. Mohiuddine ${ }^{\mathrm{a}, *}$, Kuldip Raj ${ }^{\mathrm{b}}$ \\ a Operator Theory and Applications Research Group, Department of Mathematics, Faculty of Science, King Abdulaziz University, P. O. \\ Box 80203, Jeddah 21589, Saudi Arabia. \\ ${ }^{b}$ School of Mathematics, Shri Mata Vaishno Devi University, Katra-182320, J\&K, India.
}

Communicated by M. De la Sen

\begin{abstract}
In the present paper we introduce and study vector valued Orlicz-Lorentz sequence spaces $l_{p, q, \mathcal{M}, u, \Delta, A}(X)$ on $B$ anach space $X$ with the help of a Musilak-Orlicz function $\mathcal{M}$ and for different positive indices $p$ and $q$. We also study their cross and topological duals. Finally, we introduce the operator ideals with the help of the corresponding scalar sequence spaces and s-numbers. (C)2017 All rights reserved.
\end{abstract}

Keywords: Lorentz sequence spaces, s-numbers of operators, Musielak-Orlicz function, difference sequence spaces, operator ideals. 2010 MSC: 46A45, 47B06, 47L20.

\section{Introduction and preliminaries}

Let $X$ and $Y$ be two sequence spaces and $A=\left(a_{n k}\right)$ be an infinite matrix of real or complex numbers. Then we say that $A$ defines a matrix mapping from $X$ into $Y$, if for every sequence $x=\left(x_{k}\right)_{k=0}^{\infty} \in X$, the sequence $A x=\left\{A_{n}(x)\right\}_{n=0}^{\infty}$, the $A$-transform of $x$, is in $Y$, where

$$
A_{n}(x)=\sum_{k=0}^{\infty} a_{n k} x_{k}, \quad(n \in \mathbb{N}) .
$$

By $(X, Y)$, we denote the class of all matrices $A$ such that $A: X \rightarrow Y$. Thus, $A \in(X, Y)$, if and only if the series on the right-hand side of (1.1) converges for each $n \in \mathbb{N}$ and every $x \in X$.

The matrix domain $X_{A}$ of an infinite matrix $A$ in a sequence space $X$ is defined by

$$
X_{A}=\left\{x=\left(x_{k}\right): A x \in X\right\} .
$$

The approach constructing a new sequence space by means of the matrix domain of a particular limitation method has recently been employed by several authors (see [20]).

\footnotetext{
*Corresponding author

Email addresses: mohiuddine@gmail.com (S. A. Mohiuddine), kuldipraj68@gmail .com (Kuldip Raj)
} 
The study of vector-valued sequence spaces (VVSS) was provoked by the work of Grothendieck in [6]. Since then this theory has developed considerably in different directions, (see $[3,14]$ and references given therein).

An Orlicz function $M:[0, \infty) \rightarrow[0, \infty)$ is a continuous, nondecreasing and convex function such that $M(0)=0, M(x)>0$, for $x>0$. Such function $M$ always has the integral representation

$$
M(x)=\int_{0}^{x} p(t) d t
$$

where $p(t)$, known as the kernel of $M$, is right continuous, non-decreasing function for $t>0$. It is clear that an Orlicz function $M$ is always increasing as $M(x) \rightarrow \infty$ as $x \rightarrow \infty$. Also tp $(t) \rightarrow \infty$ as $t \rightarrow \infty$ and $t p(t)=0$ for $t=0$, [11]. However $p(t)>0$ for $t=0$ is equivalent to the fact that the Orlicz sequence space $l_{M}$ is isomorphic to $l_{1}$, [8]. Therefore, we presume here that the kernel $p(t)$ has value 0 for $t=0$ and obviously $\mathrm{p}(\mathrm{t}) \rightarrow \infty$ as $\mathrm{t} \rightarrow \infty$.

For Orlicz function $M$ and kernel $p$, we define $q(s)=\sup \{t: p(t) \leqslant s\}, s \geqslant 0$. Clearly $q$ possesses the same properties as $p$ and the function $N$ defined as $N(x)=\int_{0}^{x} q(t) d t$, is an Orlicz function. The functions $M$ and $N$ are called mutually complementary functions. These functions $M$ and $N$ satisfy Young's inequality: $x y \leqslant M(x)+N(y)$, for $x y \geqslant 0$ and also $M(\alpha x) \leqslant \alpha M(x)$ for $0<\alpha<1$.

An Orlicz function $M$ is said to satisfy the $\Delta_{2}$-condition for small $x$ or at 0 , if for each $k>1$, there exist $R_{k}>0$ and $x_{k}>0$ such that

$$
M(k x) \leqslant R_{k} M(x), \text { for all } x \in\left(0, x_{k}\right] .
$$

Suppose $X$ and $Y$ are vector spaces over the same field $\mathbb{K}$ of real or complex numbers, generates a dual system $\langle X, Y\rangle$ with respect to the bilinear functional $\langle x, y\rangle$. We shall denote the vector space of all sequences formed by the elements of $X$ with respect to the operations of pointwise addition and scalar multiplication by $\Omega(X)$ and the space of all finitely non-zero sequences from $\Omega(X)$ by $\phi(X)$. A vectorvalued sequence space $\Lambda(X)$ is a subspace of $\Omega(X)$ containing $\phi(X)$. The symbol $\delta_{i}^{X}$ exists for the sequence $\{0,0, \ldots, 0, x, 0,0, \ldots\}$, where $x$ is placed at the $i$ th coordinate. The notation $\bar{x}^{(n)}$ denotes the $n$-th section of $\bar{x}$ given by $\left\{x_{1}, x_{2}, \ldots, x_{n}, 0,0, \ldots\right\}$.

A subset $M$ of $\Lambda(X)$ is said to be normal, if for $\left\{x_{i}\right\} \in M$ and $\left\{\alpha_{i}\right\} \in \mathbb{K}$, with $\left|\alpha_{i}\right| \leqslant 1, i \geqslant 1$, the sequence $\left\{\alpha_{i} x_{i}\right\} \in M$. The generalized Köthe dual of $\Lambda(X)$ is the space

$$
\Lambda^{\times}(Y)=\left\{\bar{y}=\left\{y_{i}\right\} \in Y: \sum_{i \geqslant 1}\left|\left\langle x_{i}, y_{i}\right\rangle\right|<\infty \text { for all }\left\{x_{i}\right\} \in \Lambda(X)\right\} .
$$

The generalized Köthe dual of $\Lambda^{\times}(Y)$ is denoted by $\Lambda^{\times \times}(X)$. The space $\Lambda(X)$ is said to be perfect, if $\Lambda(X)=\Lambda^{\times \times}(X)$.

A vector-valued sequence space $\Lambda(X)$ equipped with a Hausdorff locally convex topology $T$ is called

(i) a GK-space, if the maps $P_{n, \wedge(X)}: \Lambda(X) \rightarrow X, P_{n, \wedge(X)}(\bar{x})=x_{n}$, for each $n \geqslant 1$, are continuous;

(ii) a GAK-space, if $\Lambda(X)$ is a GK-space and for each $\left\{x_{i}\right\} \in \Lambda(X), \bar{x}^{(n)} \rightarrow \bar{x}$ as $n \rightarrow \infty$, in T;

(iii) a GAD-space, if $\bar{x} \in \overline{\phi(X)}$, for every $\bar{x} \in \Lambda(X)$, i.e., $\overline{\phi(X)}=\Lambda(X)$.

Remark 1.1. Every perfect sequence space $\Lambda(X)$ is normal [14].

Let us state here that if the dual system is $\left\langle X, X^{*}\right\rangle$ where $X$ is a Banach space and $X^{*}$ is its topological dual, then we may interchangeably use the notations $\langle x, f\rangle$ or $f(x)$ for $x \in X$ and $f \in X^{*}$ in the sequel.

We write $w$ for $\Omega(X), \phi$ for $\phi(X)$ and $\lambda$ for $\Lambda(X)$, if we take $X=\mathbb{K}$, the field of scalars. If $e_{\mathrm{n}}$ 's are the $n$-th unit vectors in $w$, i.e., $e^{\mathfrak{n}}=\left\{\delta_{\mathfrak{n} j}\right\}_{j=1}^{\infty}$, where $\delta_{\mathfrak{n} j}$ is the Kronecker delta, $\phi$ is clearly the subspace of $w$ spanned by $e_{n}{ }^{\prime} s, n \geqslant 1$.

A sequence space $\lambda$ is said to be symmetric, if $\bar{\alpha}_{\sigma}=\left\{\alpha_{\sigma(i)}\right\} \in \lambda$ whenever $\bar{\alpha} \in \lambda$ and $\sigma \in \Pi$, where $\Pi$ is the collection of all permutations of $\mathbb{N}$. The Köthe dual $\lambda^{\times}$of a symmetric sequence space $\lambda$ is symmetric [8]. 
The $\delta$-dual for scalar-valued sequence space $\lambda$ is defined as

$$
\lambda^{\delta}=\left\{\bar{\alpha} \in w: \sum_{i \geqslant 1}\left|\alpha_{i} \beta_{\rho(i)}\right|<\infty \text { for all } \bar{\beta} \in \lambda \text { and } \rho \in \Pi\right\} .
$$

$\lambda^{\times}$coincides with $\lambda^{\delta}$, if $\lambda$ is symmetric.

We define

$$
\lambda(X)=\left\{\left\{x_{n}\right\}: x_{n} \in X, n \geqslant 1 \text { and }\left\{\left\|x_{n}\right\|\right\} \in \lambda\right\},
$$

for a scalar-valued sequence space $\lambda$ and a Banach space $X$. In case, $\lambda$ equipped with the norm $\|\cdot\|_{\lambda}$, is a Banach space. Therefore, $\lambda(X)$ is also a Banach space with respect to the norm

$$
\|\bar{x}\|_{\lambda(X)}=\left\|\left\{\left\|x_{n}\right\|\right\}\right\|_{\lambda,} \quad(\text { see }[1,3]) .
$$

As particular cases, we have $l_{\infty}(X)$ for $\lambda=l_{\infty}$ and $c_{0}(X)$ corresponding to $\lambda=c_{0}$.

We define the set $\tilde{l}_{M}(X)$ as

$$
\tilde{\mathrm{l}}_{M}(X)=\left\{\bar{x} \in \Omega(X): \sum_{i \geqslant 1} M\left(\left\|x_{i}\right\|\right)<\infty\right\},
$$

for a Banach space $X$ corresponding to an Orlicz function $M$.

The vector-valued Orlicz sequence space is defined as

$$
l_{M}(X)=\left\{\bar{x} \in \Omega(X): \sum_{i \geqslant 1} f_{i}\left(x_{i}\right) \text { converges for all }\left\{f_{i}\right\} \in \tilde{l}_{N}\left(X^{*}\right)\right\},
$$

for mutually complementary functions $M$ and $N$.

A corresponding way of defining $l_{M}(X)$ is

$$
l_{M}(X)=\left\{\bar{x} \in \Omega(X): \sum_{i \geqslant 1} M\left(\frac{\left\|x_{i}\right\|}{\rho}\right)<\infty \text { for some } \rho>0\right\} .
$$

Two norms

$$
\|\bar{x}\|_{(M)}=\sup \left\{\left|\sum_{i \geqslant 1} f_{i}\left(x_{i}\right)\right|: \sum_{i \geqslant 1} N\left(\left\|f_{i}\right\|\right) \leqslant 1\right\},
$$

and

$$
\|\bar{x}\|_{M}=\inf \left\{\rho>0: \sum_{i \geqslant 1} M\left(\frac{\left\|x_{i}\right\|}{\rho}\right) \leqslant 1\right\},
$$

are equivalent on $l_{M}$ and hence we have

$$
\|\bar{x}\|_{M} \leqslant\|\bar{x}\|_{(M)} \leqslant 2\|\bar{x}\|_{M}, \quad \text { for } \bar{x} \in l_{M}(X), \quad \text { (see [21]). }
$$

We shall write $l_{M}(X)$ as $l_{M}$ for $X=\mathbb{K}$. If $M$ satisfies $\Delta_{2}$-condition at 0 and $M, N$ are mutually complementary Orlicz functions, then $\left(l_{M}\right)^{\times}=l_{N}[8]$.

A Musielak-Orlicz function $\mathcal{M}=\left\{M_{n}\right\}$ is a sequence of Orlicz functions (see [5, 13]). A MusielakOrlicz function $\mathcal{M}$ is said to satisfy L1 condition, if $p_{n}(x) \geqslant p_{n+1}(x)$ for all $x \in[0, \infty)$, where $p_{n}$ be the kernel of $M_{n}$, for all $n \in \mathbb{N}$. A convex modular $\rho_{\mathcal{M}}$ on $w$ for a Musielak-Orlicz function $\mathcal{M}$ is defined as

$$
\rho_{\mathcal{M}}\left(\left\{\alpha_{n}\right\}\right)=\sup _{\sigma \in \Pi} \sum_{n=1}^{\infty} M_{n}\left(\alpha_{\sigma_{(n)}}\right) .
$$

Analogous to a convex modular $\rho_{\mathcal{M}}$, we define modular space as

$$
\lambda_{\mathcal{M}}=\left\{\bar{\alpha}=\left\{\alpha_{\mathfrak{n}}\right\} \in \mathcal{w}: \rho_{\mathcal{M}}(\beta \bar{\alpha})<\infty \text {, for some } \beta>0\right\} .
$$


This space becomes a normed space under the Luxemburg norm

$$
\|\bar{\alpha}\|=\inf \left\{\beta>0: \rho_{\mathcal{M}}\left(\frac{\bar{\alpha}}{\beta}\right) \leqslant 1\right\} .
$$

A modular sequence space $\lambda_{\mathcal{M}}$ is always a symmetric sequence space.

The decreasing rearrangement of the absolute values of a sequence $\bar{\alpha}=\left\{\alpha_{n}\right\}$ in $l_{\infty}$ is given by $\left\{t_{n}(\bar{\alpha})\right\}$, where

$$
t_{\mathfrak{n}}(\bar{\alpha})=\inf \left\{\rho>0: \operatorname{card}\left\{k:\left|\alpha_{k}\right|>\rho\right\}<n\right\} .
$$

Here card $A$ denotes the cardinality of the set $A$. The sequence $\left\{t_{\mathfrak{n}}(\bar{\alpha})\right\}$ satisfies the following properties [16]:

(i) $\|\bar{\alpha}\|_{\infty}=\mathrm{t}_{1}(\bar{\alpha}) \geqslant \mathrm{t}_{2}(\bar{\alpha}) \geqslant \ldots \geqslant 0$ for $\bar{\alpha} \in \mathrm{l}_{\infty}$.

(ii) $t_{m+n-1}(\bar{\alpha}+\bar{\beta}) \leqslant t_{m}(\bar{\alpha})+t_{m}(\bar{\beta})$ for $\bar{\alpha}, \bar{\beta} \in l_{\infty}$.

(iii) $t_{m+n-1}(\bar{\alpha} \bar{\beta}) \leqslant t_{m}(\bar{\alpha}) t_{m}(\bar{\beta})$ for $\bar{\alpha}, \bar{\beta} \in l_{\infty}$.

Here $\bar{\alpha} \bar{\beta}=\left\{\alpha_{n} \beta_{n}\right\}$.

For $\bar{x}=\left\{x_{n}\right\} \in l_{\infty}(X)$, we denote by

$$
t_{n}(\bar{x})=t_{n}\left(\left\{x_{n}\right\}\right)=t_{n}\left(\left\{\left\|x_{n}\right\|\right\}\right), \quad n \in \mathbb{N} .
$$

The Lorentz sequence space $l_{p, q}(0<p, q \leqslant \infty)$ is given by

$$
l_{p, q}=\left\{\bar{\alpha}=\left\{\alpha_{n}\right\} \in l_{\infty}:\left\{n^{\frac{1}{p}-\frac{1}{q}} t_{n}(\bar{\alpha})\right\} \in l_{q}\right\} .
$$

For $\bar{\alpha} \in l_{p, q}$, let us consider the real-valued function $\|\cdot\|_{p, q}$ as follows

$$
\|\bar{\alpha}\|_{p, q}= \begin{cases}\left\{\sum_{n \geqslant 1}\left(n^{\frac{1}{p}-\frac{1}{q}} t_{n}(\bar{\alpha})\right)^{q}\right\}^{\frac{1}{q}} & \text { for } 0<q<\infty, \\ \sup _{n \geqslant 1} n^{\frac{1}{p}} t_{n}(\bar{\alpha}) & \text { for } q=\infty .\end{cases}
$$

For a convex modular $\rho_{\mathcal{M}}$ defined on $w$, it has been proved in [5], that

$$
\sum_{n \geqslant 1} M_{n}\left(t_{n}(\bar{\alpha})\right)=\rho_{\mathcal{M}}(\bar{\alpha})
$$

for $\bar{\alpha} \in \mathcal{w}$, if and only if $\mathcal{M}$ satisfies L1 condition. We see that $\left(l_{p, q},\|\cdot\|_{p, q}\right)$ is Banach spaces for $p \geqslant q$ by (1.2). But for $p<q$, it is a quasi-Banach space. Further, they are symmetric sequence spaces [15].

Throughout the paper, we shall denote the Banach spaces over the complex field $\mathbb{C}$ by $X$ and $Y$ and the class of all bounded linear maps from $X$ to $Y$ by $L(X, Y)$.

Let $L$ be the class of all bounded linear operators between any pair of Banach spaces and $w^{+}$be the class of sequences of non-negative real numbers. A mapping $s: L \rightarrow w^{+}$is called an s-number function, if it satisfies the following conditions:

(i) $\|S\|=s_{1}(S) \geqslant s_{2}(S) \geqslant \ldots \geqslant 0, s(S)=\left\{s_{n}(S)\right\}, S \in L$;

(ii) $s_{n}(S+T) \leqslant s_{n}(S)+\|T\|$ for $S, T \in L(X, Y)$ and $n \in \mathbb{N}$;

(iii) $s_{n}(R S T) \leqslant\|R\| s_{n}(S)\|T\|$ for $T \in L\left(X_{0}, X\right), S \in L(X, Y), R \in L\left(Y, Y_{0}\right)$ and $n \in \mathbb{N}$;

(iv) if rank $S<n$, then $s_{n}(S)=0$, (v), if $\operatorname{dim} X \geqslant n$, then $s_{n}\left(I_{X}\right)=1$, where $I_{X}$ denotes the identity map of $X$. 
If the condition (ii) is replaced by

(ii) ${ }^{\prime} s_{m+n-1}(S+T) \leqslant s_{m}(S)+s_{n}(T)$ for $S, T \in L(X, Y)$ and $m, n=1,2, \cdots$,

then the s-number function is called additive.

An s-number function is called multiplicative. if the condition (iii) is replaced by

(iii) ${ }^{\prime} s_{m+n-1}(R T) \leqslant s_{m}(R) s_{\mathfrak{n}}(T)$ for $R \in L\left(Y_{0}, Y\right)$ and $T \in L\left(X, Y_{0}\right), m, n=1,2, \cdots$.

We write $A(X, Y)=A \cap L(X, Y)$ for a subset $A$ of $L$. An operator ideal is a collection of $A$, if it satisfies the following:

(i) A contains all finite rank operators;

(ii) $T+S \in A(X, Y)$ for $S, T \in A(X, Y)$;

(iii) if $T \in A(X, Y)$ and $S \in L(Y, Z)$, then $S T \in A(X, Z)$ and also if $T \in L(X, Y)$ and $S \in A(Y, Z)$, then $S T \in A(X, Z)$.

For the Banach spaces $X$ and $Y$ the collection $A(X, Y)$ is called a component of $A$.

A real-valued function $f$ is said to be an ideal quasi-norm, if $f$ is defined on an operator ideal $A$ and satisfies the following properties:

(i) $0 \leqslant f(T)<\infty$, for each $T \in A$ and $f(T)=0$, if and only if $T=0$;

(ii) there exists a constant $\sigma \geqslant 1$ such that $f(S+T) \leqslant \sigma[f(S)+f(T)]$ for $S, T \in A(X, Y)$, where $A(X, Y)$ is any component of $A$;

(iii) (a) $f(R S) \leqslant\|R\| f(S)$, for $S \in A(X, Z), R \in L(Z, Y)$, and

(b) $f(R S) \leqslant\|S\| f(R)$, for $S \in L(X, Z), R \in A(Z, Y)$.

An operator ideal is said to be quasi-normed operator ideal, if it is equipped with an ideal quasi-norm and a quasi-Banach operator ideal is a quasi-normed operator ideal of which each component is complete with respect to the ideal quasi-norm.

The notion of difference sequence spaces was introduced by Kizmaz [10] who studied the difference sequence spaces $l_{\infty}(\Delta), c(\Delta)$ and $c_{0}(\Delta)$. The notion was further generalized by Et and Çolak [4] by introducing the spaces $l_{\infty}\left(\Delta^{\mathrm{m}}\right), \mathrm{c}\left(\Delta^{\mathrm{m}}\right)$ and $\mathrm{c}_{0}\left(\Delta^{\mathrm{m}}\right)$. Let $\mathrm{m}$ be a non-negative integer, then for $Z=c, c_{0}$ and $l_{\infty}$, we have sequence spaces

$$
\mathrm{Z}\left(\Delta^{\mathrm{m}}\right)=\left\{x=\left(\mathrm{x}_{\mathrm{k}}\right) \in \mathcal{w}:\left(\Delta^{\mathrm{m}} \mathrm{x}_{\mathrm{k}}\right) \in \mathrm{Z}\right\},
$$

where $\Delta^{\mathrm{m}} \mathrm{x}=\left(\Delta^{\mathrm{m}} \mathrm{x}_{\mathrm{k}}\right)=\left(\Delta^{\mathrm{m}-1} \mathrm{x}_{\mathrm{k}}-\Delta^{\mathrm{m}-1} \mathrm{x}_{\mathrm{k}+1}\right)$ and $\Delta^{0} \mathrm{x}_{\mathrm{k}}=\mathrm{x}_{\mathrm{k}}$ for all $\mathrm{k} \in \mathbb{N}$, which is equivalent to the following binomial representation

$$
\Delta^{\mathrm{m}} \mathrm{x}_{\mathrm{k}}=\sum_{\nu=0}^{\mathrm{m}}(-1)^{v}\left(\begin{array}{c}
\mathrm{m} \\
v
\end{array}\right) \mathrm{x}_{\mathrm{k}+v} .
$$

Taking $\mathrm{m}=1$, we get the spaces studied by Et and Çolak [4]. For more details about this work one can refer to $[1,2,9,12,16-18]$.

\section{The vector-valued sequence spaces $l_{p, q, \mathcal{M}, u, \Delta, A}(X)$ and $h_{p, q, \mathcal{M}, u, \Delta, A}(X)$}

Let $X$ be a Banach space. Let $\mathcal{M}=\left(M_{k}\right)$ be an Musielak-Orlicz function, that is, $\mathcal{M}$ is a sequence of Orlicz functions, $u=\left(u_{k}\right)$ be a sequence of strictly positive real numbers and $A=\left(a_{n k}\right)$ be a nonnegative 
two-dimensional bounded-regular matrix. In this paper we define the following classes of sequences:

$$
\begin{aligned}
& \mathrm{l}_{p, q, \mathcal{M}, u, \Delta, A}(X)=\left\{\bar{x}=\left\{x_{k}\right\} \in \mathrm{l}_{\infty}(X): \sum_{k \geqslant 1} u_{k}\left[M_{k}\left(\frac{\left\|A k^{\frac{1}{p}-\frac{1}{q}} t_{k}\left(\Delta^{m} x_{k}\right)\right\|}{\rho}\right)\right]<\infty, \text { for some } \rho>0\right\}, \\
& h_{p, q, \mathcal{M}, u, \Delta, A}(X)=\left\{\bar{x}=\left\{x_{k}\right\} \in l_{\infty}(X): \sum_{k \geqslant 1} u_{k}\left[M_{k}\left(\frac{\left\|A k^{\frac{1}{p}-\frac{1}{q}} t_{k}\left(\Delta^{m} x_{k}\right)\right\|}{\delta}\right)\right]<\infty, \text { for all } \delta>0\right\} .
\end{aligned}
$$

For $\bar{x} \in l_{p, q, \mathcal{M}, u, \Delta, A}(X)$, we define

$$
\|\bar{x}\|_{p, q, \mathcal{M}, u, \Delta, A}(X)=\inf \left\{\rho>0: \sum_{k \geqslant 1} u_{k}\left[M_{k}\left(\frac{\left\|A k^{\frac{1}{p}-\frac{1}{q}} t_{k}\left(\Delta^{m} \chi_{k}\right)\right\|}{\rho}\right)\right] \leqslant 1\right\} .
$$

If we take $\mathcal{M}(x)=x$ in $l_{p, q, \mathcal{M}, u, \Delta, A}(X)$ and $h_{p, q, \mathcal{M}, u, \Delta, A}(X)$, then we have the following spaces:

$$
\begin{aligned}
& \mathrm{l}_{p, q, u, \Delta, A}(X)=\left\{\bar{x}=\left\{x_{k}\right\} \in l_{\infty}(X): \sum_{k \geqslant 1} u_{k}\left[\frac{\left\|A k^{\frac{1}{p}-\frac{1}{q}} t_{k}\left(\Delta^{m} x_{k}\right)\right\|}{\rho}\right]<\infty, \text { for some } \rho>0\right\}, \\
& h_{p, q, u, \Delta, A}(X)=\left\{\bar{x}=\left\{x_{k}\right\} \in l_{\infty}(X): \sum_{k \geqslant 1} u_{k}\left[\frac{\left\|A k^{\frac{1}{p}-\frac{1}{q}} t_{k}\left(\Delta^{m} x_{k}\right)\right\|}{\delta}\right]<\infty, \text { for all } \delta>0\right\} .
\end{aligned}
$$

Let $u=\left(u_{k}\right)=1$, for all $k \in \mathbb{N}$. Then the spaces $l_{p, q, \mathcal{M}, u, \Delta, A}(X)$ and $h_{p, q, \mathcal{M}, u, \Delta, A}(X)$ are reduced to $l_{p, q, \mathcal{M}, \Delta, A}(X)$ and $h_{p, q, \mathcal{M}, \Delta, A}(X)$, respectively, as follow:

$$
\begin{aligned}
& l_{p, q, \mathcal{M}, \Delta, A}(X)=\left\{\bar{x}=\left\{x_{k}\right\} \in l_{\infty}(X): \sum_{k \geqslant 1}\left[M_{k}\left(\frac{\left\|A k^{\frac{1}{p}-\frac{1}{q}} t_{k}\left(\Delta^{m} x_{k}\right)\right\|}{\rho}\right)\right]<\infty, \text { for some } \rho>0\right\}, \\
& h_{p, q, \mathcal{M}, \Delta, A}(X)=\left\{\bar{x}=\left\{x_{k}\right\} \in l_{\infty}(X): \sum_{k \geqslant 1}\left[M_{k}\left(\frac{\left\|A k^{\frac{1}{p}-\frac{1}{q}} t_{k}\left(\Delta^{m} x_{k}\right)\right\|}{\delta}\right)\right]<\infty, \text { for all } \delta>0\right\} .
\end{aligned}
$$

If we take $A=(C, 1)$ in $l_{p, q, \mathcal{M}, u, \Delta, A}(X)$ and $h_{p, q, \mathcal{M}, u, \Delta, A}(X)$, then we have the following spaces:

$$
\begin{aligned}
& \mathrm{l}_{\mathrm{p}, \mathrm{q}, \mathcal{M}, \mathrm{u}, \Delta}(X)=\left\{\bar{x}=\left\{\mathrm{x}_{\mathrm{k}}\right\} \in \mathrm{l}_{\infty}(X): \sum_{\mathrm{k} \geqslant 1} \mathfrak{u}_{\mathrm{k}}\left[\mathrm{M}_{\mathrm{k}}\left(\frac{\left\|\mathrm{k}^{\frac{1}{p}-\frac{1}{q}} \mathrm{t}_{\mathrm{k}}\left(\Delta^{\mathrm{m}} \mathrm{x}_{\mathrm{k}}\right)\right\|}{\rho}\right)\right]<\infty \text {, for some } \rho>0\right\} \text {, } \\
& \mathrm{h}_{\mathrm{p}, \mathrm{q}, \mathcal{M}, \mathrm{u}, \Delta}(X)=\left\{\bar{x}=\left\{\mathrm{x}_{\mathrm{k}}\right\} \in \mathrm{l}_{\infty}(\mathrm{X}): \sum_{\mathrm{k} \geqslant 1} \mathfrak{u}_{\mathrm{k}}\left[\mathrm{M}_{\mathrm{k}}\left(\frac{\left\|\mathrm{k}^{\frac{1}{p}-\frac{1}{q}} \mathrm{t}_{\mathrm{k}}\left(\Delta^{\mathrm{m}} \mathrm{x}_{\mathrm{k}}\right)\right\|}{\delta}\right)\right]<\infty, \text { for all } \delta>0\right\} \text {. }
\end{aligned}
$$

If we take $A=(C, 1)$ and $\mathcal{M}(x)=x$ in $l_{p, q, \mathcal{M}, u, \Delta, A}(X)$ and $h_{p, q, \mathcal{M}, u, \Delta, A}(X)$, then we have the following spaces:

$$
\begin{aligned}
& \mathrm{l}_{p, q, u, \Delta}(X)=\left\{\bar{x}=\left\{x_{k}\right\} \in l_{\infty}(X): \sum_{k \geqslant 1} u_{k}\left[\frac{\left\|k^{\frac{1}{p}-\frac{1}{q}} t_{k}\left(\Delta^{m} x_{k}\right)\right\|}{\rho}\right]<\infty, \quad \text { for some } \rho>0\right\}, \\
& h_{p, q, u, \Delta,}(X)=\left\{\bar{x}=\left\{x_{k}\right\} \in l_{\infty}(X): \sum_{k \geqslant 1} u_{k}\left[\frac{\left\|k^{\frac{1}{p}-\frac{1}{q}} t_{k}\left(\Delta^{m} x_{k}\right)\right\|}{\delta}\right]<\infty, \text { for all } \delta>0\right\} .
\end{aligned}
$$

If we take $\left(M_{k}\right)=M, A=I,\left(u_{k}\right)=1$ for all $k \in \mathbb{N}$ and $m=0$, then we get the analogous of the spaces defined by Gupta and Bhar [7]. The aim of this paper is to study the vector-valued OrliczLorentz sequence spaces. We also study their structural properties and investigate cross and topological duals of these spaces. Finally we prove that the operator ideals defined with the help of scalar-valued sequence spaces $l_{p, q, \mathcal{M}, u, \Delta, A}$ and additive s-numbers are quasi-Banach operator ideals for $p<q$ and Banach operator ideals for $p \geqslant q$. 
Theorem 2.1. Let $\mathcal{M}=\left(\mathrm{M}_{\mathrm{k}}\right)$ be a Musielak-Orlicz function, $\mathrm{u}=\left(\mathrm{u}_{\mathrm{k}}\right)$ be a sequence of strictly positive real numbers and $\mathrm{A}=\left(\mathrm{a}_{\mathrm{nk}}\right)$ be a nonnegative two-dimensional bounded-regular matrix. Then the space $\mathrm{l}_{\mathrm{p}, \mathrm{q}, \mathcal{M}, \mathrm{u}, \Delta, \mathrm{A}}(\mathrm{X})$ equipped with $\|\cdot\|_{p, q, \mathcal{M}, u, \Delta, A}$ is a quasi-Banach space for $\mathrm{p}<\mathrm{q}$ and Banach space for $\mathrm{p} \geqslant \mathrm{q}$. Further for $\bar{x} \in l_{p, q}, \mathcal{M}, u, \Delta, A(X)$, we have

$$
\sum_{k \geqslant 1} \mathfrak{u}_{k}\left[M_{k}\left(\frac{\left\|A k^{\frac{1}{p}-\frac{1}{q}} t_{k}\left(\Delta^{m} \chi_{k}\right)\right\|}{\|\bar{x}\|_{p, q}, \mathcal{M}, u, \Delta, A}\right)\right] \leqslant 1 .
$$

Proof. We can easily show that $l_{p, q, \mathcal{M}, u, \Delta, A}(X)$ is a vector space with usual coordinate wise addition and scalar multiplication. To show that $\|\cdot\|_{p, q, \mathcal{M}, u, \Delta, A}$ is a quasi-norm, let $\|\bar{x}\|_{p, q, \mathcal{M}, u, \Delta, A} \geqslant 0$, for each $\bar{x} \in l_{p, q, \mathcal{M}, u, \Delta, A}(X)$ and $\|\bar{x}\|_{p, q, \mathcal{M}, u, \Delta, A}=0$, for $\bar{x}=0$. Suppose that $\|\bar{x}\|_{p, q, \mathcal{M}, u, \Delta, A}=0$, for some $\bar{x}=$ $\left\{x_{k}\right\} \in l_{p, q, \mathcal{M}, u, \Delta, A}(X)$ and for given $\varepsilon>0$, we can find $\rho>0$ such that $\rho<\varepsilon$ and

$$
\sum_{k \geqslant 1} u_{k}\left[M_{k}\left(\frac{\left\|A k^{\frac{1}{p}-\frac{1}{q}} t_{k}\left(\Delta^{m} x_{k}\right)\right\|}{\rho}\right)\right] \leqslant 1 .
$$

When $\bar{x} \neq 0$, we get $\left\|x_{k_{0}}\right\| \neq 0$ for some $k_{0} \in \mathbb{N}$ and so $t_{k_{1}}\left(\Delta^{m} x_{k}\right)=\left\|\Delta^{m} x_{k_{0}}\right\|$, for some $k_{1} \in \mathbb{N}$ implies

$$
u_{k}\left[M_{k}\left(\frac{\left\|A k_{1}^{\frac{1}{p}-\frac{1}{q}} t_{k_{1}}\left(\Delta^{m} x_{k}\right)\right\|}{\varepsilon}\right)\right] \leqslant u_{k}\left[M_{k}\left(\frac{\left\|A k_{1}^{\frac{1}{p}-\frac{1}{q}} t_{k_{1}}\left(\Delta^{m} x_{k}\right)\right\|}{\rho}\right)\right] \leqslant 1,
$$

for any $\varepsilon>0$. We get a contradiction to the fact, so $\bar{x}=0$.

To prove triangular-type inequality, let us consider $\bar{x}=\left\{x_{k}\right\}$ and $\bar{y}=\left\{y_{k}\right\} \in l_{p, q, \mathcal{M}, u, \Delta, A}(X)$. Thus for any $\varepsilon>0$, there exist $\rho_{1}, \rho_{2}>0$ such that

$$
\rho_{1}<\|\bar{x}\|_{p, q, \mathcal{M}, u, \Delta, A}+\frac{\varepsilon}{2} \text { with } \sum_{k \geqslant 1} \mathfrak{u}_{k}\left[M_{k}\left(\frac{\left\|A k^{\frac{1}{p}-\frac{1}{q}} t_{k}\left(\Delta^{m} x_{k}\right)\right\|}{\rho_{1}}\right)\right] \leqslant 1,
$$

and

$$
\rho_{2}<\|\bar{y}\|_{p, q, \mathcal{M}, u, \Delta, A}+\frac{\varepsilon}{2} \text { with } \sum_{k \geqslant 1} \mathfrak{u}_{k}\left[M_{k}\left(\frac{\left\|A k^{\frac{1}{p}-\frac{1}{q}} t_{k}\left(\Delta^{m} y_{k}\right)\right\|}{\rho_{2}}\right)\right] \leqslant 1 .
$$

If $\frac{1}{p}-\frac{1}{q}>0$, then via properties (i) and (ii) of $\left\{t_{k}\left(\Delta^{m} x_{k}\right)\right\}$, we get

$$
\begin{aligned}
& \sum_{k \geqslant 1} u_{k}\left[M _ { k } \left(\frac{\left\|A k^{\frac{1}{p}-\frac{1}{q}} t_{k}\left(\Delta^{m}\left(x_{k}+y_{k}\right)\right)\right\|}{\left.2^{\frac{1}{p}-\frac{1}{q}+1}\left(\rho_{1}+\rho_{2}\right)\right]=} \sum_{k \geqslant 1} u_{k}\left[M_{k}\left(\frac{\left\|A(2 k)^{\frac{1}{p}-\frac{1}{q}} t_{2 k}\left(\Delta^{m}\left(x_{k}+y_{k}\right)\right)\right\|}{2^{\frac{1}{p}-\frac{1}{q}+1}\left(\rho_{1}+\rho_{2}\right)}\right)\right]\right.\right. \\
&+\sum_{k \geqslant 1} u_{k}\left[M_{k}\left(\frac{\left\|A(2 k-1)^{\frac{1}{p}-\frac{1}{q}} t_{2 k-1}\left(\Delta^{m}\left(x_{k}+y_{k}\right)\right)\right\|}{2^{\frac{1}{p}-\frac{1}{q}+1}\left(\rho_{1}+\rho_{2}\right)}\right)\right] \\
& \leqslant 2 \sum_{k \geqslant 1} u_{k}\left[M_{k}\left(\frac{\left\|A k^{\frac{1}{p}-\frac{1}{q}}\left(t_{k}\left(\Delta^{m} x_{k}\right)+t_{k}\left(\Delta^{m} y_{k}\right)\right)\right\|}{2\left(\rho_{1}+\rho_{2}\right)}\right)\right] \\
& \leqslant \sum_{k \geqslant 1} u_{k}\left[M_{k}\left(\frac{\rho_{1}}{\rho_{1}+\rho_{2}}\right)\left(\frac{\left\|A k^{\frac{1}{p}-\frac{1}{q}} t_{k}\left(\Delta^{m_{2}} x_{k}\right)\right\|}{\rho_{1}}\right)\right. \\
&\left.+\left(\frac{\rho_{2}}{\rho_{1}+\rho_{2}}\right)\left(\frac{\left\|A k^{\frac{1}{p}-\frac{1}{q}} t_{k}\left(\Delta^{m} y_{k}\right)\right\|}{\rho_{2}}\right)\right] \\
& \leqslant 1 .
\end{aligned}
$$

Hence,

$$
\|\bar{x}+\bar{y}\|_{p, q, \mathcal{M}, u, \Delta, A} \leqslant 2^{\frac{1}{p}-\frac{1}{q}+1}\left(\rho_{1}+\rho_{2}\right)
$$




$$
\leqslant 2^{\frac{1}{p}-\frac{1}{q}+1}\left(\|\bar{x}\|_{p, q, \mathcal{M}, u, \Delta, A}+\|\bar{y}\|_{p, q, \mathcal{M}, u, \Delta, A}+\varepsilon\right) .
$$

Now we prove the completeness of the space $\left(l_{p, q, \mathcal{M}, u, \Delta, A}(X),\|\cdot\|_{p, q, \mathcal{M}, u, \Delta, A}\right)$. Let $\left\{\bar{x}_{k}\right\}$ be a Cauchy sequence in $l_{p, q, \mathcal{M}, u, \Delta, A}(X)$, as $\bar{x}_{k}=\left\{x_{k}^{\mathfrak{n}}\right\}_{n} \geqslant 1, k \in \mathbb{N}$. Hence for $\varepsilon>0$, there exists $k_{0} \in \mathbb{N}$ such that

$$
\left\|\bar{x}_{k+j}-\bar{x}_{k}\right\|_{p, q, \mathcal{M}, u, \Delta, A}=\inf \left\{\rho>0: \sum_{n \geqslant 1} u_{n}\left[M_{n}\left(\frac{\left\|A n^{\frac{1}{p}-\frac{1}{q}} t_{n}\left(\Delta^{m}\left(\bar{x}_{k+j}-\bar{x}_{k}\right)\right)\right\|}{\rho}\right)\right] \leqslant 1\right\}<\varepsilon,
$$

for each $k \geqslant k_{0}$ and each $j \in \mathbb{N}$. Thus

$$
\sum_{n \geqslant 1}\left[\frac{\left\|A n^{\frac{1}{p}-\frac{1}{q}} t_{n}\left(\Delta^{m}\left(\bar{x}_{k+j}-\bar{x}_{k}\right)\right)\right\|}{\varepsilon}\right] \leqslant 1, \quad \text { for all } k \geqslant k_{0}, j \in \mathbb{N},
$$

which implies that

$$
\left\{\left\{A n^{\frac{1}{p}-\frac{1}{q}} t_{n}\left(\Delta^{m}\left(\bar{x}_{k+j}-\bar{x}_{k}\right)\right) / \varepsilon\right\}: n \in \mathbb{N}\right\},
$$

is a bounded set for $j \in \mathbb{N}$ and for all $k \geqslant k_{0}$. Therefore $\left\{x_{k}^{n}\right\}$ is a Cauchy sequence in $X$, for each $n \in \mathbb{N}$ and so converges to $z_{n}$. Let $\bar{z}=\left\{z_{n}\right\}$. Then $t_{n}\left(\Delta^{m}\left(\bar{x}_{k+j}-\bar{x}_{k}\right)\right) \rightarrow t_{n}\left(\bar{z}-\bar{x}_{k}\right)$ as $j \rightarrow \infty$ and hence by continuity of $\mathcal{M}$,

$$
\sum_{n \geqslant 1} u_{n}\left[M_{n}\left(\frac{\left\|A n^{\frac{1}{p}-\frac{1}{q}} t_{n}\left(\Delta^{m}\left(\bar{z}-\bar{x}_{k}\right)\right)\right\|}{\varepsilon}\right)\right] \leqslant 1, \text { for all } k \geqslant k_{0} .
$$

This implies that $\bar{z} \in l_{p, q, \mathcal{M}, u, \Delta, A}(X)$ and $\left\|\bar{z}-\bar{x}_{k}\right\|_{p, q, \mathcal{M}, u, \Delta, A} \rightarrow 0$ as $k \rightarrow \infty$. Also, inequality (2.1) is directly obtained from the definition of the quasi-norm $\|\cdot\|_{p, q, \mathcal{M}, u, \Delta, A}$. This completes the proof.

Theorem 2.2. Let $\mathcal{M}=\left(\mathrm{M}_{\mathrm{k}}\right)$ be a Musielak-Orlicz function, $\mathrm{u}=\left(\mathrm{u}_{\mathrm{k}}\right)$ be a sequence of strictly positive real numbers and $\mathrm{A}=\left(\mathrm{a}_{\mathrm{nk}}\right)$ be a nonnegative two-dimensional bounded-regular matrix. Then $\mathrm{h}_{\mathrm{p}, \mathrm{q}, \mathcal{M}, \mathrm{u}, \Delta, \mathrm{A}}(\mathrm{X})$ is a closed subspace of $l_{p, q, \mathcal{M}, u, \Delta, A}(X)$. Moreover, if $\mathcal{M}=\left(M_{k}\right)$ satisfies $\Delta_{2}$-condition at 0 , then $l_{p, q, \mathcal{M}, u, \Delta, A}(X)=$ $h_{p, q, \mathcal{M}, u, \Delta, A}(X)$.

Proof. First of all it is without a doubt that $h_{p, q, \mathcal{M}, u, \Delta, A}(X)$ is a subspace of $l_{p, q, \mathcal{M}, u, \Delta, A}(X)$. Now we

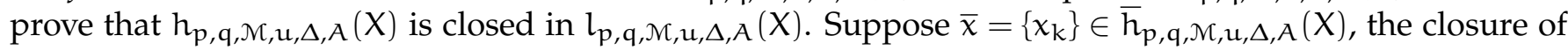

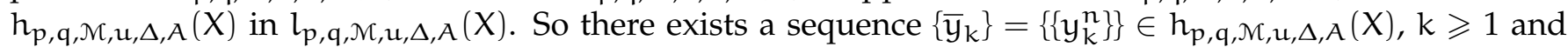
we have $\left\|\bar{y}_{k}-\bar{x}\right\|_{p, q}, \mathcal{M}, u, \Delta, A \rightarrow 0$ as $k \rightarrow \infty$. Take any $\delta>0$. Thus for $\delta_{1}=\min \left\{2^{\frac{1}{p}-\frac{1}{q}} \delta\right.$, $\left.\delta\right\}$, we get $k_{0} \in \mathbb{N}$ such that

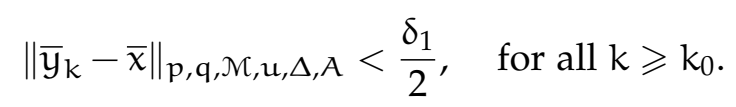

When $\frac{1}{p}-\frac{1}{q} \geqslant 0$, we have

$$
\begin{aligned}
\sum_{n \geqslant 1} u_{n}\left[M_{n}\left(\frac{\left\|A n^{\frac{1}{p}-\frac{1}{q}} t_{n}\left(\Delta^{m} \bar{x}\right)\right\|}{\delta}\right)\right] \leqslant & 2 \sum_{n \geqslant 1} u_{n}\left[M_{n}\left(\frac{\left\|A n^{\frac{1}{p}-\frac{1}{q}}\left(t_{n}\left(\Delta^{m}\left(\bar{x}-\bar{y}_{k_{0}}\right)\right)+t_{n}\left(\Delta^{m} \bar{y}_{k_{0}}\right)\right)\right\|}{\delta_{1}}\right)\right] \\
\leqslant & \sum_{n \geqslant 1} u_{n}\left[M_{n}\left(\frac{\left\|A n^{\frac{1}{p}-\frac{1}{q}}\left(t_{n}\left(\Delta^{m}\left(\bar{x}-\bar{y}_{k_{0}}\right)\right)\right)\right\|}{\delta_{1} / 2}\right)\right] \\
& +\sum_{n \geqslant 1} u_{n}\left[M_{n}\left(\frac{\left\|A n^{\frac{1}{p}-\frac{1}{q}} t_{n}\left(\Delta^{m} \bar{y}_{k_{0}}\right)\right\|}{\delta_{1} / 2}\right)\right] \\
\leqslant & \sum_{n \geqslant 1} u_{n}\left[M_{n}\left(\frac{\left\|A n^{\frac{1}{p}-\frac{1}{q}}\left(t_{n}\left(\Delta^{m}\left(\bar{x}-\bar{y}_{k_{0}}\right)\right)\right)\right\|}{\left\|\bar{x}-\bar{y}_{k_{0}}\right\|_{p, q, \mathcal{M}, u, \Delta, A}}\right)\right]
\end{aligned}
$$




$$
\begin{aligned}
& +\sum_{n \geqslant 1} u_{n}\left[M_{n}\left(\frac{\left\|A n^{\frac{1}{p}-\frac{1}{q}} t_{n}\left(\Delta^{m} \bar{y}_{k_{0}}\right)\right\|}{\delta_{1} / 2}\right)\right] \\
& <\infty
\end{aligned}
$$

In the case when $\frac{1}{p}-\frac{1}{q}<0$, we get

$$
\begin{aligned}
\sum_{n \geqslant 1} u_{n}\left[M_{n}\left(\frac{\left\|A n^{\frac{1}{p}-\frac{1}{q}} t_{n}\left(\Delta^{m} \bar{x}\right)\right\|}{\delta}\right)\right] \leqslant & \sum_{n \geqslant 1} u_{n}\left[M_{n}\left(\frac{\left\|A n^{\frac{1}{p}-\frac{1}{q}}\left(t_{n}\left(\Delta^{m}\left(\bar{x}-\bar{y}_{k_{0}}\right)\right)\right)\right\|}{\delta_{1} / 2}\right)\right] \\
& +\sum_{n \geqslant 1} u_{n}\left[M_{n}\left(\frac{\left\|A n^{\frac{1}{p}-\frac{1}{q}} t_{n}\left(\Delta^{m} \bar{y}_{k_{0}}\right)\right\|}{\delta_{1} / 2}\right)\right] \\
& <\infty,
\end{aligned}
$$

by the relation (2.2). Clearly $\bar{x} \in h_{p, q, \mathcal{M}, u, \Delta, A}(X)$ and so the subspace $h_{p, q, \mathcal{M}, u, \Delta, A}(X)$ is closed. Now we suppose that $\mathcal{M}$ satisfies $\Delta_{2}$-condition at 0 . Let $\bar{x} \in l_{p, q, \mathcal{M}, u, \Delta, A}(X)$, we have

$$
\sum_{k \geqslant 1} u_{k}\left[M_{k}\left(\frac{\left\|A k^{\frac{1}{p}-\frac{1}{q}} t_{k}\left(\Delta^{m} x_{k}\right)\right\|}{\rho_{0}}\right)\right]<\infty, \quad \text { for some } \rho_{0}>0
$$

To show that $\bar{x} \in h_{p, q, \mathcal{M}, u, \Delta, A}(X)$, choose any $\eta>0$. If $\eta \geqslant \rho_{0}$, then

$$
\sum_{k \geqslant 1} u_{k}\left[M_{k}\left(\frac{\left\|A k^{\frac{1}{p}-\frac{1}{q}} t_{k}\left(\Delta^{m} x_{k}\right)\right\|}{\eta}\right)\right]<\infty
$$

Now presume $\eta<\rho_{0}$ and suppose $K=\frac{\rho_{0}}{\eta}$. Since $\mathcal{M}$ satisfies the $\Delta_{2}$-condition, so we can find $R_{K}>0$ and $x_{K}>0$ such that $\mathcal{M}(K x) \leqslant R_{K} \mathcal{M}(x)$ for all $x \in\left(0, x_{K}\right]$

$$
\Longrightarrow \sum_{k \geqslant k_{0}} u_{k}\left[M_{k}\left(\frac{\left\|K A k^{\frac{1}{p}-\frac{1}{q}} t_{k}\left(\Delta^{m} x_{k}\right)\right\|}{\rho_{0}}\right)\right] \leqslant R_{K} \sum_{k \geqslant k_{0}} u_{k}\left[M_{k}\left(\frac{\left\|A k^{\frac{1}{p}-\frac{1}{q}} t_{k}\left(\Delta^{m} x_{k}\right)\right\|}{\rho_{0}}\right)\right]<\infty,
$$

for some $k_{0} \in \mathbb{N}$. Hence

$$
\sum_{k \geqslant 1} u_{k}\left[M_{k}\left(\frac{\left\|A k^{\frac{1}{p}-\frac{1}{q}} t_{k}\left(\Delta^{m} \chi_{k}\right)\right\|}{\eta}\right)\right]<\infty, \quad \text { for any } \eta>0,
$$

and so $\bar{x} \in h_{p, q, \mathcal{M}, u, \Delta, A}(X)$, we have $h_{p, q, \mathcal{M}, u, \Delta, A}(X)=l_{p, q, \mathcal{M}, u, \Delta, A}(X)$.

Proposition 2.3. Let $\mathcal{M}=\left(\mathrm{M}_{\mathrm{k}}\right)$ be a Musielak-Orlicz function, $\mathrm{u}=\left(\mathrm{u}_{\mathrm{k}}\right)$ be a sequence of strictly positive real numbers and $\mathrm{A}=\left(\mathrm{a}_{\mathrm{nk}}\right)$ be a nonnegative two-dimensional bounded-regular matrix. If $\mathrm{Y}=\mathrm{h}_{\mathrm{p}, \mathrm{q}, \mathcal{M}, \mathrm{u}, \Delta, \mathrm{A}}(\mathrm{X}) \cap$ $\mathrm{c}_{0}(\mathrm{X}), 0<\mathrm{p}, \mathrm{q} \leqslant \infty$. Then $\mathrm{Y}$ equipped with the subspace topology of $\mathrm{h}_{\mathrm{p}, \mathrm{q}, \mathcal{M}, \mathrm{u}, \Delta, \mathrm{A}}(\mathrm{X})$ is a GAD-space.

Proof. Obviously $\phi(X) \subset Y$. Suppose $\bar{x} \in Y$. Now for any $\varepsilon>0$, we can find $k_{0} \in \mathbb{N}$, such that

$$
\sum_{k \geqslant k_{0}} u_{k}\left[M_{k}\left(\frac{\left\|A k^{1 / p-1 / q} t_{k}\left(\Delta^{m} x_{k}\right)\right\|}{\varepsilon}\right)\right] \leqslant 1
$$

Let $\mathrm{I}_{k}=\left\{i \in \mathbb{N}:\left\|x_{i}\right\|>\frac{1}{k}\right\}, k \in \mathbb{N}$ and $\bar{v}_{k}=\sum_{i \in I_{k}} \delta_{i}^{x_{i}}$. Since $\bar{x} \in c_{0}(X), I_{k}$ is finite and so $\bar{v}_{k} \in \phi(X)$. Set $n_{k}=$ card $I_{k}$. Then take $m_{0} \in \mathbb{N}$ such that

$$
\sum_{k \geqslant m_{0}} u_{k}\left[M_{k}\left(\frac{\left\|A k^{1 / p-1 / q} t_{k}\left(\Delta^{m} x_{k}\right)\right\|}{\varepsilon}\right)\right] \leqslant \frac{1}{2} .
$$


Take $k$ so large that

$$
\frac{1}{k} \sum_{i=1}^{m_{0}} u_{k}\left[M_{k}\left(\frac{\left\|A i^{1 / p-1 / q}\right\|}{\varepsilon}\right)\right] \leqslant \frac{1}{2}
$$

Thus,

$$
\begin{aligned}
\sum_{i \geqslant 1} u_{k}\left[M_{k}\left(\frac{\left\|A i^{1 / p-1 / q} t_{n_{k}+i}\left(\Delta^{m} x_{k}\right)\right\|}{\varepsilon}\right)\right] \leqslant & \frac{1}{k} \sum_{i=1}^{m_{0}} u_{k}\left[M_{k}\left(\frac{\left\|A i^{1 / p-1 / q}\right\|}{\varepsilon}\right)\right] \\
& +\sum_{i \geqslant m_{0}+1} u_{k}\left[M_{k}\left(\frac{\left\|A k^{1 / p-1 / q} t_{i}\left(\Delta^{m} x_{k}\right)\right\|}{\varepsilon}\right)\right] \\
& \leqslant \frac{1}{2}+\frac{1}{2}=1 .
\end{aligned}
$$

This implies that $\left\|\bar{x}-\bar{v}_{k}\right\|_{p, q, \mathcal{M}, u, \Delta, A} \leqslant \varepsilon$, for sufficiently large $k$. Hence $Y$ is a GAD-space.

Proposition 2.4. Let $\mathcal{M}=\left(M_{k}\right)$ be a Musielak-Orlicz function satisfying $\Delta_{2}$-condition at $0, \mathfrak{u}=\left(\mathfrak{u}_{\mathrm{k}}\right)$ be a sequence of strictly positive real numbers and $\mathrm{A}=\left(\mathrm{a}_{\mathrm{nk}}\right)$ be a nonnegative two-dimensional bounded-regular matrix. If $\mathrm{l}_{\mathrm{p}, \mathrm{q}, \mathcal{M}, \mathrm{u}, \Delta, \mathrm{A}}(\mathrm{X}) \subset \mathrm{c}_{0}(\mathrm{X}), 0<\mathrm{p} \leqslant \mathrm{q} \leqslant \infty$. Then $\mathrm{l}_{\mathrm{p}, \mathrm{q}, \mathcal{M}, \mathrm{u}, \Delta, \mathrm{A}}$ is a GAD-space.

Remark 2.5. It is very motivating to know whether the space $h_{p, q, \mathcal{M}, u, \Delta, A}(X)$ is a GAK-space, this means that the $k^{\text {th }}$ section $\bar{x}^{(k)}=\left\{x_{1}, x_{2}, \ldots, x_{k}, 0,0,0,0, \ldots\right\}$ of an element $\bar{x}=\left\{x_{i}\right\}$ of $h_{p, q, \mathcal{M}, u, \Delta, A}$ converges to $\bar{x}$ with respect to its quasi-norm. Whenever, if $p, q>0$ with $\frac{1}{p}-\frac{1}{q} \geqslant 0$ and $\bar{x} \in h_{p, q, M}, u, \Delta, A(X)$ such that $\left\|x_{1}\right\|>\left\|x_{2}\right\|>\left\|x_{3}\right\|>\cdots$, then $t_{k}(\bar{x})=\left\|x_{k}\right\|$ and in this case, one can easily show that $\left\|\bar{x}-\bar{x}^{(k)}\right\|_{p, q, \mathcal{M}, u, \Delta, A} \rightarrow 0$ as $k \rightarrow \infty$.

\section{Duals of the space $l_{p, q, \mathcal{M}, u, \Delta, A}(X), 1 \leq p \leq q \leq \infty$}

Suppose that the spaces $l_{p, q, \mathcal{M}, u, \Delta, A}(X)$ are symmetric sequence spaces, since the decreasing rearrangement of $\bar{x}$ would be the same as that of $\bar{x}_{\pi}$ for any permutation $\pi$ of $\mathbb{N}$ and $\mathcal{M}=\left(M_{k}\right)$ is an increasing function. Thus the $\delta$-dual of the scalar-valued sequence space $l_{p, q, \mathcal{M}, u, \Delta, A}$ would coincide with its cross-dual.

Theorem 3.1. Let $\mathcal{M}=\left(\mathrm{M}_{\mathrm{k}}\right)$ and $\mathcal{N}=\left(\mathrm{N}_{\mathrm{k}}\right)$ be two mutually complementary Musielak-Orlicz functions such that $\mathcal{M}$ satisfies $\Delta_{2}$-condition at $0, \mathrm{u}=\left(\mathrm{u}_{\mathrm{k}}\right)$ be a sequence of strictly positive real numbers and $\mathrm{A}=\left(\mathrm{a}_{\mathrm{nk}}\right)$ be a nonnegative two-dimensional bounded-regular matrix. Then $\left(l_{p_{1}, q_{1}, \mathcal{A}, u, \Delta, A}\right)^{\times} \supseteq l_{p_{2}, q_{2}, \mathcal{N}, u, \Delta, A}$, where $1 / p_{1}+$ $1 / p_{2}=1$ and $1 / q_{1}+1 / q_{2}=1$. Moreover, $\left(l_{p_{1}, q_{1}, \mathcal{M}, u, \Delta, A}\right)^{\times}=l_{p_{2}, q_{2}, \mathcal{N}, u, \Delta, A}$ when $1 / p_{1}-1 / q_{1} \geqslant 0$.

Proof. To show that $l_{p_{2}, q_{2}, \mathcal{N}, u, \Delta, A} \subset\left(l_{p_{1}, q_{1}, \mathcal{M}, u, \Delta, A}\right)^{\times}$, suppose $\bar{\beta} \in l_{p_{2}, q_{2}, \mathcal{N}, u, \Delta, A}$. Then, we have

$$
\sum_{k \geqslant 1} u_{k}\left[N_{k}\left(\frac{\left\|A k^{\frac{1}{p_{2}}-\frac{1}{q_{2}}} t_{k}\left(\Delta^{m} \bar{\beta}\right)\right\|}{\delta_{0}}\right)\right]<\infty, \quad \text { for some } \delta_{0}>0
$$

Let $\bar{\alpha} \in \mathrm{l}_{\mathfrak{p}_{1}, \mathrm{q}_{1}, \mathcal{M}, \mathrm{u}, \Delta, A}$. Then $\sum_{\mathrm{k} \geqslant 1} \mathfrak{u}_{\mathrm{k}}\left[\mathrm{M}_{\mathrm{k}}\left(\frac{\left\|A k^{\frac{1}{p_{1}}-\frac{1}{q_{1}}} t_{k}\left(\Delta^{m} \bar{\alpha}\right)\right\|}{\rho}\right)\right]<\infty, \quad$ for all $\rho>0$. Thus,

$$
\begin{aligned}
\sum_{k \geqslant 1}\left|\alpha_{k} \beta_{k}\right| & \leqslant \sum_{k \geqslant 1} t_{k}\left(\Delta^{m} \bar{\alpha}\right) t_{k}\left(\Delta^{m} \bar{\beta}\right) \\
& \leqslant \sum_{k \geqslant 1} \mathfrak{u}_{k}\left[M_{k}\left(\frac{\left\|A k^{\frac{1}{p_{1}}-\frac{1}{q_{1}}} t_{k}\left(\Delta^{m} \bar{\alpha}\right)\right\|}{1 / \delta_{0}}\right)\right]+\sum_{k \geqslant 1} \mathfrak{u}_{k}\left[N_{k}\left(\frac{\left\|A k^{\frac{1}{p_{2}}-\frac{1}{q_{2}}} t_{k}\left(\Delta^{m} \bar{\beta}\right)\right\|}{\delta_{0}}\right)\right]<\infty .
\end{aligned}
$$


Hence $\bar{\beta} \in\left(l_{p_{1}, q_{1}, \mathcal{M}, u, \Delta, A}\right)^{\times}$. Now to prove $\left(l_{p_{1}, q_{1}, \mathcal{M}, u, \Delta, A}\right)^{\times}=l_{p_{2}, q_{2}, \mathcal{N}, u, \Delta, A}$, suppose $\bar{\beta} \in\left(l_{p_{1}, q_{1}, \mathcal{M}, u, \Delta, A}\right)^{\times}$, then $\sum_{i \geqslant 1}\left|\alpha_{i} \beta_{i}\right|<\infty$, for all $\left\{\alpha_{i}\right\} \in l_{p_{1}, q_{1}, \mathcal{M}, u, \Delta, A}$. Since $l_{p_{1}, q_{1}, \mathcal{M}, u, \Delta, A}$ and $\left(l_{p_{1}, q_{1}, \mathcal{M}, u, \Delta, A}\right)^{\times}$both are sym-

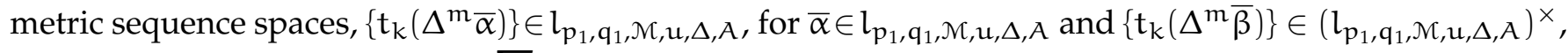
for $\bar{\beta} \in\left(l_{p_{1}, q_{1}, \mathcal{M}, u, \Delta, A}\right)^{\times}$. Hence $\sum_{k \geqslant 1} t_{k}\left(\Delta^{m} \bar{\alpha}\right) t_{k}\left(\Delta^{m} \bar{\beta}\right)<\infty$, for all $\bar{\alpha} \in l_{p_{1}, q_{1}, \mathcal{M}, u, \Delta, A}$.

Again if $\bar{\gamma} \in l_{\mathcal{M}}$, then $\left\{t_{k}\left(\Delta^{m} \bar{\gamma}\right)\right\} \in l_{\mathcal{M}}$ as $l_{\mathcal{M}}$ is symmetric and normal and so

$$
\left\{A k^{\frac{1}{p_{2}}-\frac{1}{q_{2}}} t_{k}\left(\Delta^{m} \bar{\gamma}\right)\right\} \in l_{p_{1}, q_{1}, \mathcal{M}, u, \Delta, A} .
$$

Hence

$$
\sum_{k \geqslant 1}\left[\left\|A k^{\frac{1}{p_{2}}-\frac{1}{q_{2}}} t_{k}\left(\Delta^{m} \bar{\gamma}\right) t_{k}\left(\Delta^{m} \bar{\beta}\right)\right\|\right]<\infty, \quad \text { for all } \bar{\gamma} \in l_{\mathcal{M}}
$$

This implies that $\left\{A k^{\frac{1}{p_{2}}-\frac{1}{q_{2}}} t_{k}\left(\Delta^{m} \bar{\beta}\right)\right\} \in l_{\mathcal{M}}^{\times}=l_{\mathcal{N}}$ and so $\bar{\beta} \in l_{p_{2}, q_{2}, \mathcal{N}, u, \Delta, A}$. Thus, we have

$$
\left(l_{p_{1}, q_{1}, \mathcal{M}, u, \Delta, A}\right)^{\times}=l_{p_{2}, q_{2}, \mathcal{N}, u, \Delta, A} .
$$

Proposition 3.2. Let $\mathcal{M}=\left(\mathrm{M}_{\mathrm{k}}\right)$ be a Musielak-Orlicz function, $\mathrm{u}=\left(\mathrm{u}_{\mathrm{k}}\right)$ be a sequence of strictly positive real numbers and $\mathrm{A}=\left(\mathrm{a}_{\mathrm{nk}}\right)$ be a nonnegative two-dimensional bounded-regular matrix. For positive reals $\mathrm{p}_{1}, \mathrm{p}_{2}, \mathrm{q}_{1}, \mathrm{q}_{2}$ with $1 / p_{1}+1 / p_{2}=1,1 / q_{1}+1 / q_{2}=1$ such that $q_{1}<p_{1}$, the spaces $l_{p_{1}, q_{1}, \mathcal{M}, u, \Delta, A}$ are perfect sequences spaces.

Proof. In fact in this case $\left(l_{p_{2}, q_{2}, \mathcal{N}, u, \Delta, A}\right)^{\times}=l_{p_{1}, q_{1}, \mathcal{M}, u, \Delta, A}$ and $l_{p_{2}, q_{2}, \mathcal{N}, u, \Delta, A} \subseteq\left(l_{p_{1}, q_{1}, \mathcal{M}, u, \Delta, A}\right)^{\times}$. So we have $l_{p_{1}, q_{1}, \mathcal{M}, u, \Delta, A} \subset\left(l_{p_{1}, q_{1}, \mathcal{M}, u, \Delta, A}\right)^{\times \times} \subset\left(l_{p_{2}, q_{2}, N, u, \Delta, A}\right)^{\times}=l_{p_{1}, q_{1}, \mathcal{M}, u, \Delta, A}$.

Proposition 3.3. Let $\mathcal{M}=\left(M_{k}\right)$ be an Musielak-Orlicz function satisfying $\Delta_{2}$-condition at $0, u=\left(u_{k}\right)$ be a sequence of strictly positive real numbers and $A=\left(a_{n k}\right)$ be a nonnegative two-dimensional bounded-regular matrix. Let $X$ be a Banach space and $p_{1}, p_{2}, q_{1}, q_{2}$ are such that $1 / p_{1}+1 / p_{2}=1,1 / q_{1}+1 / q_{2}=1$ and $1 / p_{1}-1 / q_{1}>0$. Then $\left(l_{p_{1}, q_{1}, \mathcal{M}, u, \Delta, A}(X)\right)^{\times}=l_{p_{2}, q_{2}, \mathcal{N}, u, \Delta, A}\left(X^{*}\right)$.

Proof. One can easily prove it by using Theorem 3.1, so we omit the proof.

Theorem 3.4. Let $\mathcal{M}=\left(\mathrm{M}_{\mathrm{k}}\right)$ be a Musielak-Orlicz function, $\mathrm{u}=\left(\mathrm{u}_{\mathrm{k}}\right)$ be a sequence of strictly positive real numbers and $\mathrm{A}=\left(\mathrm{a}_{\mathrm{nk}}\right)$ be a nonnegative two-dimensional bounded-regular matrix. Suppose $\mathrm{p}_{1}, \mathrm{p}_{2}, \mathrm{q}_{1}, \mathrm{q}_{2}$ are real numbers with $1<\mathrm{p}_{1}, \mathrm{q}_{1}, \mathrm{p}_{2}, \mathrm{q}_{2}<\infty$ and $1 / \mathrm{p}_{1}+1 / \mathrm{p}_{2}=1,1 / \mathrm{q}_{1}+1 / \mathrm{q}_{2}=1$. Then the dual of $\mathrm{l}_{\mathrm{p}_{1}, \mathrm{q}_{1}, \mathcal{M}, \mathrm{u}, \Delta, A}(X)$ is topologically isomorphic to $l_{p_{2}, q_{2}, \mathcal{N}, u, \Delta, A}\left(X^{*}\right)$, if and only if the sequence $\left\{f_{i}\right\} \in l_{p_{2}, q_{2}, N, u, \Delta, A}\left(X^{*}\right)$ is identified with the linear functional $\mathrm{F}$ given by

$$
F\left(\left\{x_{i}\right\}\right)=\sum_{i \geqslant 1}\left\langle x_{i}, f_{i}\right\rangle, \text { for each }\left\{x_{i}\right\} \in l_{p_{1}, q_{1}, \mathcal{M}, u, \Delta, A}(X) .
$$

Proof. Subsequently for $\left\{f_{i}\right\} \in l_{p_{2}, q_{2}, \mathcal{N}, u, \Delta, A}\left(X^{*}\right)$, we define a linear functional $F$ on $l_{p_{1}, q_{1}, \mathcal{A}, u, \Delta, A}(X)$ as in $(+)$ where convergence of the series is being guaranteed by Proposition 3.3. For $k \in \mathbb{N}$, let

$$
F_{k}\left(\left\{x_{i}\right\}\right)=\sum_{i=1}^{k}\left\langle x_{i}, f_{i}\right\rangle, \quad\left\{x_{i}\right\} \in l_{p_{1}, q_{1}, \mathcal{M}, u, \Delta, A}(X) .
$$

Obviously, $\left\{F_{k}\right\}$ is a sequence of continuous linear functionals on $l_{p_{1}, q_{1}, \mathcal{M}, u, \Delta, A}(X)$ converging pointwise to $F$. Thus $F$ is continuous by Banach-Steinhaus Theorem (see [19]). Hence, $F \in\left(l_{p_{1}, q_{1}, \mathcal{M}, u, \Delta, A}(X)\right)^{*}$. Next, for $\bar{x} \in l_{p_{1}, q_{1}, \mathcal{M}, u, \Delta, A}(X)$, we get 


$$
\begin{aligned}
|\mathbf{f} \overline{(x)}| & \leqslant \sum_{i \geqslant 1}\left|\left\langle x_{i}, f_{i}\right\rangle\right| \\
& \leqslant \sum_{i \geqslant 1} t_{i}(\bar{x}) t_{i}(\bar{f}) \\
& \leqslant\|\bar{f}\|_{p_{2}, q_{2}, \mathcal{N}, u, \Delta, A} \sum_{i \geqslant 1} u_{i}\left[M _ { i } \left(\mid A\left(i^{1 / p_{1}-1 / q_{1}} t_{i}\left(\Delta^{m} \bar{x}\right)\right) \| \frac{\left\|\left(i^{1 / p_{2}-1 / q_{2}} t_{i}\left(\Delta^{m} \bar{f}\right)\right)\right\|}{\left.\left.\|\bar{f}\|_{p_{2}, q_{2}, \mathcal{N}, u, \Delta, A}\right)\right]}\right.\right. \\
& \leqslant\|\bar{f}\|_{p_{2}, q_{2}, \mathcal{N}, u, \Delta, A}\left\|u_{i} A\left\{i^{1 / p_{1}-1 / q_{1}} t_{i}\left(\Delta^{m} \bar{x}\right)\right\}\right\|_{(\mathcal{M})},
\end{aligned}
$$

since $\sum_{i \geqslant 1} u_{i}\left[N_{i}\left(\frac{\left\|A i^{1 / p_{2}-1 / q_{2}} t_{i}\left(\Delta^{m} \bar{f}\right)\right\|}{\|\bar{f}\|_{p_{2}, q_{2}, \mathcal{N}, u, \Delta, A}}\right)\right] \leqslant 1$. Therefore,

$$
|\mathrm{F}(\overline{\mathrm{x}})| \leqslant 2\|\overline{\mathrm{f}}\|_{\mathrm{p}_{2}, \mathbf{q}_{2}, \mathcal{N}, u, \Delta, A}\|\bar{x}\|_{p_{1}, \mathbf{q}_{1}, \mathcal{M}, u, u, \Delta, A},
$$

for any $\bar{x} \in l_{p_{1}, q_{1}, \mathcal{M}, u, \Delta, A}(X)$. Thus,

$$
\|f\| \leqslant 2\left\|\left\{f_{i}\right\}\right\|_{p_{2}, q_{2}, \mathcal{N}, u, \Delta, A} .
$$

Conversely, suppose $F \in\left(l_{p_{1}, q_{1}, \mathcal{M}, u, \Delta, A}(X)\right)^{*}$. Define $f_{i} \in X^{*}, i \in \mathbb{N}$ as $f_{i}(x)=F\left(\delta_{i}^{x}\right)$. Now to prove $\left\{f_{i}\right\} \in l_{p_{2}, q_{2}, \mathcal{N}, u, \Delta, A}\left(X^{*}\right)$ we choose $\left\{\alpha_{i}\right\} \in l_{p_{1}, q_{1}, \mathcal{M}, u, \Delta, A}$. Take $\left\{x_{i}\right\} \subseteq X$ with $\left\|x_{i}\right\|=1$ and $\left\|f_{i}\right\|<f_{i}\left(x_{i}\right)+$ $1 / 2^{i}$, for all $i \in \mathbb{N}$. Let $\left\{\beta_{i}\right\} \subset \mathbb{C}$ be such that $\left|f_{i}\left(\alpha_{i} x_{i}\right)\right|=f_{i}\left(\alpha_{i} \beta_{i} x_{i}\right)$, for all $i \in \mathbb{N}$. Obviously, $\left|\beta_{i}\right|=1$, for all $i \in \mathbb{N}$, and so $\left\{\alpha_{i} \beta_{i} x_{i}\right\} \in l_{p_{1}, q_{1}, \mathcal{M}, u, \Delta, A}(X)$. Suppose

$$
\begin{aligned}
\sum_{i \geqslant 1}\left|\alpha_{i}\right|\left\|f_{i}\right\| & <\sum_{i \geqslant 1} f_{i}\left(\alpha_{i} \beta_{i} x_{i}\right)+\sum_{i \geqslant 1} \frac{\alpha_{i}}{2^{i}} \\
& =\sum_{i \geqslant 1} F\left(\delta^{\alpha_{i} \beta_{i} x_{i}}\right)+K \\
& =\lim _{k \rightarrow \infty} \sum_{i=1}^{k} F\left(\delta^{\alpha_{i} \beta_{i} x_{i}}\right)+K \\
& =F\left(\left\{\delta^{\alpha_{i} \beta_{i} x_{i}}\right\}\right)+K,
\end{aligned}
$$

where $K=\sum_{i \geqslant 1} \frac{\alpha_{i}}{2^{i}}$. Therefore, $\sum_{i \geqslant 1}\left|\alpha_{i}\right|\left\|f_{i}\right\|<\infty$, for all $\left\{\alpha_{i}\right\} \in l_{p_{1}, q_{1}, \mathcal{M l}, u, \Delta, A}$ and hence $\left\{f_{i}\right\} \in l_{p_{2}, q_{2}, \mathcal{N}, u, \Delta, A}\left(X^{*}\right)$ by Theorem 3.1. To prove that $F$ has the form as given in equation (3.1), suppose for $\left\{x_{i}\right\} \in l_{p_{1}, q_{1}, \mathcal{M}, u, \Delta, A}(X)$

$$
\begin{aligned}
\sum_{i \geqslant 1}\left|\left\langle x_{i}, f_{i}\right\rangle\right| & =\sum_{i \geqslant 1}\left|F\left(\delta^{x_{i}}\right)\right| \\
& =\lim _{k \rightarrow \infty} \sum_{i=1}^{k} F\left(\delta^{\beta_{i} x_{i}}\right)=F\left(\left\{\beta_{i} x_{i}\right\}\right),
\end{aligned}
$$

where $\beta_{i}$ are taken as above. Thus $\sum_{i \geqslant 1}\left|\left\langle x_{i}, f_{i}\right\rangle\right|<\infty$. Hence,

$$
\sum_{i \geqslant 1}\left|\left\langle x_{i}, f_{i}\right\rangle\right| \text { is unconditionally convergent. }
$$

Now if $0<p_{1}<q_{1} \leqslant \infty, l_{p_{1}, q_{1}, \mathcal{M}, u, \Delta, A}(X)$ is a GAD-space. We write $t_{i}(\bar{x})=\left\|x_{\phi}(i)\right\|$, for some $\phi \in \pi$ and $\bar{u}_{k}=\sum_{i=1}^{k} \delta_{\phi_{(i)}}^{x_{\phi_{(i)}}}$, for $k \in \mathbb{N}$. Therefore $\bar{u}_{k} \in \phi(X)$ and $\left\|\bar{x}-\bar{u}_{k}\right\|_{p_{1}, q_{1}, \mathcal{M}, u, \Delta, A} \rightarrow 0$ as $k \rightarrow \infty$ by Proposition 3.3. Then,

$$
F\left(\left\{x_{i}\right\}\right)=F\left(\lim _{k \rightarrow \infty} \bar{u}_{k}\right)=\sum_{i \geqslant 1}\left\langle x_{i}, f_{i}\right\rangle
$$


by (3.3). Hence the mapping $R: l_{p_{2}, q_{2}, \mathcal{N}, u, \Delta, A}\left(X^{*}\right) \rightarrow\left(l_{p_{1}, q_{1}, \mathcal{M}, u, \Delta, A}(X)\right)^{*}$ defined by $R(\bar{f})=F$, with $\bar{f}=\left\{f_{i}\right\}, f_{i(x)}=F\left(\delta_{i}^{x}\right), i \in \mathbb{N}$ is a topological isomorphism from equations (3.1), (3.2) and the open mapping theorem (see [19]). This completes the proof.

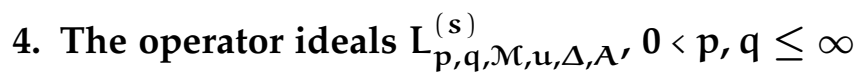

Let $\mathcal{M}=\left(M_{k}\right)$ be a Musielak-Orlicz function and $X, Y$ are Banach spaces.

Definition 4.1. Let $T: X \rightarrow Y$ be a bounded linear operator. Then $T$ is said to be of type $l_{p, q, \mathcal{M}, u, \Delta, A}$, if $\left\{s_{k}(T)\right\} \in l_{p, q, \mathcal{M}, u, \Delta, A}$. We shall denote the set of all above mappings by $L_{p, q}^{(s)}, \mathcal{M}, u, \Delta, A$ where

$$
\mathrm{L}_{\mathrm{p}, \mathrm{q}, \mathcal{M}, \mathrm{M}, \Delta, \mathrm{A}, \mathrm{A}}^{(\mathrm{s})}=\left\{\mathrm{T} \in \mathrm{L}:\left\{\mathrm{s}_{\mathrm{k}}(\mathrm{T})\right\} \in \mathrm{l}_{\mathrm{p}, \mathrm{q}, \mathcal{M}, \mathrm{M}, \Delta, \mathrm{A}, \mathrm{A}} .\right.
$$

We define the norm for any $T \in \mathrm{L}_{\mathrm{p}, \mathrm{q}, \mathcal{M}, \mathrm{M}, \mathrm{u}, \Delta, \mathrm{A}}^{(\mathrm{s})}$ as

$$
\|T\|_{p, q, \mathcal{M}, u, \Delta, A}=\inf \left\{\rho>0: \sum_{k \geqslant 1} u_{k}\left[M_{k}\left(\frac{\left\|A k^{\frac{1}{p}-\frac{1}{q}} \Delta^{m} s_{k}(T)\right\|}{\rho}\right)\right] \leqslant 1\right\} .
$$

Theorem 4.2. Let $\mathcal{M}=\left(\mathrm{M}_{\mathrm{k}}\right)$ be a Musielak-Orlicz function, $\mathrm{u}=\left(\mathrm{u}_{\mathrm{k}}\right)$ be a sequence of strictly positive real numbers and $\mathrm{A}=\left(\mathrm{a}_{\mathrm{nk}}\right)$ be a nonnegative two-dimensional bounded-regular matrix. Then for $\mathrm{p}<\mathrm{q}, \mathrm{L}_{\mathrm{p}, \mathrm{q}, \mathrm{M}, \mathrm{u}, \Delta, \mathrm{A}}$ equipped with $\|\cdot\|_{\mathrm{p}, \mathrm{q}, \mathfrak{M}, \mathrm{u}, \Delta, \mathrm{A}}$ is a quasi-Banach operator ideal and for $\mathrm{p} \geqslant \mathrm{q}$ it is a Banach ideal.

Proof. To show that $\mathrm{L}_{\mathrm{p}, \mathrm{q}, \mathcal{M}, u, \Delta, A}^{(s)}$ is an operator ideal, firstly note that all finite rank operators are contained

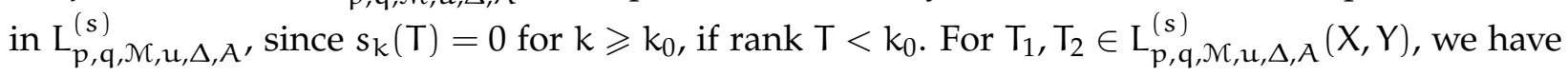

$$
\sum_{k \geqslant 1} \mathfrak{u}_{k}\left[M_{k}\left(\frac{\left\|A k^{\frac{1}{p}-\frac{1}{q}} \Delta^{m} s_{k}\left(T_{1}\right)\right\|}{\rho_{1}}\right)\right]<\infty
$$

and

$$
\sum_{k \geqslant 1} \mathfrak{u}_{k}\left[M_{k}\left(\frac{\left\|A k^{\frac{1}{p}-\frac{1}{q}} \Delta^{m} s_{k}\left(T_{2}\right)\right\|}{\rho_{2}}\right)\right]<\infty,
$$

for some $\rho_{1}, \rho_{2}>0$. Firstly, we consider the condition when $\frac{1}{p}-\frac{1}{q} \geqslant 0$

$$
\begin{aligned}
\sum_{k \geqslant 1} u_{k}\left[M_{k}\left(\frac{\left\|A k^{\frac{1}{p}-\frac{1}{q}} \Delta^{m} s_{k}\left(T_{1}+T_{2}\right)\right\|}{2^{\frac{1}{p}-\frac{1}{q}+1}\left(\rho_{1}+\rho_{2}\right)}\right)\right] \leqslant & \sum_{k \geqslant 1} \frac{\rho_{1}}{\rho_{1}+\rho_{2}} u_{k}\left[M_{k}\left(\frac{\left\|A k^{\frac{1}{p}-\frac{1}{q}} \Delta^{m} s_{k}\left(T_{1}\right)\right\|}{\rho_{1}}\right)\right] \\
& +\sum_{k \geqslant 1} \frac{\rho_{2}}{\rho_{1}+\rho_{2}} u_{k}\left[M_{k}\left(\frac{\left\|A k^{\frac{1}{p}-\frac{1}{q}} \Delta^{m} s_{k}\left(T_{2}\right)\right\|}{\rho_{2}}\right)\right] \\
& <\infty .
\end{aligned}
$$

Again, if $\frac{1}{p}-\frac{1}{q}<0$, then

$$
\begin{aligned}
\sum_{k \geqslant 1} \mathfrak{u}_{k}\left[M_{k}\left(\frac{\left\|A k^{\frac{1}{p}-\frac{1}{q}} \Delta^{m} s_{k}\left(T_{1}+T_{2}\right)\right\|}{\left(\rho_{1}+\rho_{2}\right)}\right)\right] \leqslant & \sum_{k \geqslant 1} \frac{\rho_{1}}{\rho_{1}+\rho_{2}} u_{k}\left[M_{k}\left(\frac{\left\|A k^{\frac{1}{p}-\frac{1}{q}} \Delta^{m} s_{k}\left(T_{1}\right)\right\|}{\rho_{1}}\right)\right] \\
& +\sum_{k \geqslant 1} \frac{\rho_{2}}{\rho_{1}+\rho_{2}} \mathfrak{u}_{k}\left[M_{k}\left(\frac{\left\|A k^{\frac{1}{p}-\frac{1}{q}} \Delta^{m} s_{k}\left(T_{2}\right)\right\|}{\rho_{2}}\right)\right] \\
& <\infty .
\end{aligned}
$$

This implies that $T_{1}+T_{2} \in L_{p, q, \mathcal{M}, u, \Delta, A}^{(s)}(X, Y)$. Now, we want to show that for $T \in L_{p, q, \mathcal{M}, u, \Delta, A}^{(s)}(E, F)$, 
$R \in L(F, Y)$ and $S \in L(X, E), R T S \in L_{p, q, \mathcal{M}, u, \Delta, A}^{(s)}(X, Y)$. Thus for $T \in L_{p, q, \mathcal{M}, u, \Delta, A}^{(s)}(E, F)$, we have

$$
\sum_{k \geqslant 1} u_{k}\left[M_{k}\left(\frac{\left\|A k^{\frac{1}{p}-\frac{1}{q}} \Delta^{m} s_{k}(T)\right\|}{\rho_{0}}\right)\right]<\infty,
$$

for some $\rho_{0}>0$ and hence

$$
\sum_{k \geqslant 1} \mathfrak{u}_{k}\left[M_{k}\left(\frac{\left\|A k^{\frac{1}{p}-\frac{1}{q}} \Delta^{m} s_{k}(R T S)\right\|}{\|R\|\|S\| \rho_{0}}\right)\right]<\infty,
$$

by the property (iii) of s-number function. Thus $R T S \in L_{p, q, \mathcal{M}, u, \Delta, A}^{(s)}(X, Y)$. Therefore, $L_{p, q, \mathcal{M}, u, \Delta, A}^{(s)}$ is an operator ideal.

The proof of the function $\|\cdot\|_{p, q, \mathcal{M}, u, \Delta, A}$ is a quasi-norm (or, norm) defined on $\mathrm{L}_{p, q, \mathcal{M}, u, \Delta, A}^{(s)}$ is similar to one defined on $L_{p, q, \mathcal{M}, u, \Delta, A}(X)$ and so excluded. To prove the completeness, suppose $\left\{T_{k}\right\}$ is a Cauchy sequence in component of $\mathrm{L}_{p, q, \mathcal{M}, u, \Delta, A}^{(s)}(X, Y)$ of $\mathrm{L}_{p, q, \mathcal{M}, u, \Delta, A}^{(s)}$. Thus for $\varepsilon>0$, there exists $k_{0} \in \mathbb{N}$ such that

$$
\left\|T_{k+j}-T_{k}\right\|_{p, q, \mathcal{M}, u, \Delta, A}<\varepsilon, \quad \text { for all } k \geqslant k_{0} \text { and } j \in \mathbb{N} \text {. }
$$

This implies that there exists $\rho>0$ such that $\rho<\varepsilon$ and

$$
\sum_{n \geqslant 1} u_{n}\left[M_{n}\left(\frac{\left\|A n^{\frac{1}{p}-\frac{1}{q}} \Delta^{m} s_{n}\left(T_{k+j}-T_{k}\right)\right\|}{\varepsilon}\right)\right] \leqslant 1, \text { for all } k \geqslant k_{0}, j \in \mathbb{N} \text {. }
$$

Thus,

$$
\left\{\frac{A n^{\frac{1}{p}-\frac{1}{q}} \Delta^{m} s_{n}\left(T_{k+j}-T_{k}\right)}{\varepsilon} ; n \geqslant 1\right\},
$$

is a bounded sequence for each $k \geqslant k_{0}$ and $j \in \mathbb{N}$. Therefore for some constant $K>0$, we get

$$
\left\|T_{k+j}-T_{k}\right\|<\varepsilon K, \quad \text { for all } k \geqslant k_{0}, j \in \mathbb{N} \text {. }
$$

Thus, $\left\{T_{k}\right\}$ is a Cauchy sequence in $L(X, Y)$. So there exists a $T \in L(X, Y)$ such that $\left\|T_{k}-T\right\| \rightarrow 0$ as $k \rightarrow \infty$. As $s_{n}\left(T_{k}-T\right) \leqslant\left\|T_{k}-T\right\|$, for all $k \geqslant 1$, we have $s_{n}\left(T_{k}-T\right) \rightarrow 0$ as $k \rightarrow \infty$. Also,

$$
\left|s_{n}\left(T_{k+j}-T_{k}\right)-s_{n}\left(T-T_{k}\right)\right| \leqslant\left\|T_{k+j}-T_{k}\right\|,
$$

which implies

$$
s_{n}\left(T_{k+j}-T_{k}\right) \rightarrow s_{n}\left(T-T_{k}\right) \text { as } j \rightarrow \infty
$$

Now, we have from (4.1),

$$
\sum_{n \geqslant 1} u_{n}\left[M_{n}\left(\frac{\left\|A n^{\frac{1}{p}-\frac{1}{q}} \Delta^{m} s_{n}\left(T-T_{k}\right)\right\|}{\varepsilon}\right)\right] \leqslant 1, \text { for all } k \geqslant k_{0} .
$$

This implies that $T-\mathrm{T}_{k} \in \mathrm{L}_{\mathrm{p}, \mathrm{q}, \mathcal{M}, \mathfrak{u}, \Delta, A}^{(s)}(X, Y)$ and $\left\|\mathrm{T}-\mathrm{T}_{k}\right\|_{p, q, \mathcal{M}, u, \Delta, A}<\varepsilon$, for all $k \geqslant k_{0}$. Therefore, $\mathrm{T} \in \mathrm{L}_{\mathrm{p}, \mathbf{q}, \mathcal{M}, \mathrm{u}, \Delta, A}^{(\mathrm{s})}$ and $\mathrm{T}_{\mathrm{k}} \rightarrow \mathrm{T} \in \mathrm{L}_{\mathbf{p}, \mathbf{q}, \mathcal{M}, \mathrm{u}, \Delta, A}^{(s)}$, which shows that $\mathrm{L}_{\mathbf{p}, \mathbf{q}, \mathcal{M}, \mathrm{u}, \Delta, A}^{(s)}$ is a quasi-Banach operator ideal.

Theorem 4.3. Let $\mathcal{N}=\left(\mathrm{M}_{\mathrm{k}}\right)$ and $\mathcal{N}=\left(\mathrm{N}_{\mathrm{k}}\right)$ be two complementary Musielak-Orlicz functions, $u=\left(\mathrm{u}_{\mathrm{k}}\right)$ be a sequence of strictly positive real numbers, $A=\left(\mathrm{a}_{\mathrm{nk}}\right)$ be a nonnegative two-dimensional bounded-regular matrix and $\mathrm{s}$ is a multiplicative s-number function. If $0<\mathrm{p}_{1}, \mathrm{p}_{2}, \mathrm{p}, \mathrm{q}_{1}, \mathrm{q}_{2}, \mathrm{q}<\infty$ are such that $\frac{1}{\mathrm{p}_{1}}+\frac{1}{\mathrm{p}_{2}}=\frac{1}{\mathrm{p}^{\prime}}$, $\frac{1}{q_{1}}+\frac{1}{q_{2}}=\frac{1}{q_{1}}$, then

$$
\mathrm{L}_{\mathrm{p}_{1}, \mathbf{q}_{1}, \mathcal{M}, \mathrm{u}, \Delta, \mathrm{A}}^{(\mathrm{s})} \circ \mathrm{L}_{\mathrm{p}_{2}, \mathrm{q}_{2}, \mathcal{N}, \mathrm{u}, \Delta, \mathrm{A}}^{(\mathrm{s})} \subset \mathrm{L}_{\mathrm{p}, \mathrm{q}, 1, \mathrm{u}, \Delta, \mathrm{A}^{\prime}}^{(s)}
$$


where

$$
\mathrm{L}_{p, q, 1, u, \Delta, A}^{(s)}=\left\{\mathrm{T} \in \mathrm{L}: \sum_{\mathrm{k} \geqslant 1} \mathfrak{u}_{k}\left[\left\|A k^{\frac{1}{p}-\frac{1}{q}} \Delta^{m} s_{k} \mathrm{~T}\right\|\right] \in \mathrm{l}_{1}\right\} .
$$

Proof. Suppose $T \in \mathrm{L}_{\mathrm{p}_{1}, \mathrm{q}_{1}, \mathcal{M}, u, \Delta, \Delta, A}^{(s)} \circ \mathrm{L}_{\mathrm{p}_{2}, \mathrm{q}_{2}, \mathcal{N}, \mathrm{u}, \Delta, A}^{(s)}(X, Y)$. Then $\mathrm{T}=\mathrm{T}_{1} \mathrm{~T}_{2}$, where $\mathrm{T}_{1} \in \mathrm{L}_{\mathrm{p}_{1}, \mathrm{q}_{1}, \mathcal{M}, \mathrm{M}, \mathrm{u}, \Delta, A}^{(s)}(\mathrm{Z}, \mathrm{Y})$ and $\mathrm{T}_{2} \in \mathrm{L}_{p_{2}, \mathrm{q}_{2}, \mathcal{N}, u, \Delta, A}^{(s)}(X, Z)$ and here $Z$ is a Banach space. Thus

$$
\begin{aligned}
& \sum_{k \geqslant 1} u_{k}\left[M_{k}\left(\frac{\left\|A k^{\frac{1}{p_{1}}-\frac{1}{q_{1}}} \Delta^{m} s_{k}\left(T_{1}\right)\right\|}{\rho_{1}}\right)\right]<\infty, \text { for some } \rho_{1}>0, \\
& \sum_{k \geqslant 1} u_{k}\left[N_{k}\left(\frac{\left\|A k^{\frac{1}{p_{2}}-\frac{1}{q_{2}}} \Delta^{m} s_{k}\left(T_{2}\right)\right\|}{\rho_{2}}\right)\right]<\infty, \text { for some } \rho_{2}>0 .
\end{aligned}
$$

If $\frac{1}{p}-\frac{1}{q} \geqslant 0$, we have

$$
\begin{aligned}
\sum_{k} \geqslant 1 & u_{k}\left[\frac{\left\|A k^{\frac{1}{p}-\frac{1}{q}} \Delta^{m} s_{k}\left(T_{1} T_{2}\right)\right\|}{2^{\frac{1}{p}-\frac{1}{q}} \rho_{1} \rho_{2}}\right] \\
& \leqslant 2 \sum_{k \geqslant 1} u_{k}\left[M_{k}\left(\frac{\left\|A k^{\frac{1}{p_{1}}-\frac{1}{q_{1}}} \Delta^{m^{m}} s_{k}\left(T_{1}\right)\right\|}{\rho_{1}}\right)\right]+\sum_{k \geqslant 1} u_{k}\left[N_{k}\left(\frac{\left\|A k^{\frac{1}{p_{2}}-\frac{1}{q_{2}}} \Delta^{m} s_{k}\left(T_{2}\right)\right\|}{\rho_{2}}\right)\right]<\infty,
\end{aligned}
$$

and for $\frac{1}{p}-\frac{1}{q}<0$, we have

$$
\begin{aligned}
& \sum_{k \geqslant 1} u_{k}\left[\frac{\left\|A k^{\frac{1}{p}-\frac{1}{q}} \Delta^{m} s_{k}\left(T_{1} T_{2}\right)\right\|}{\rho_{1} \rho_{2}}\right] \leqslant 2 \sum_{k \geqslant 1} u_{k}\left[M_{k}\left(\frac{\left\|A k^{\frac{1}{p_{1}}-\frac{1}{q_{1}}} \Delta^{m_{1}} s_{k}\left(T_{1}\right)\right\|}{\rho_{1}}\right)\right] \\
&+\sum_{k \geqslant 1} u_{k}\left[N_{k}\left(\frac{\left\|A k^{\frac{1}{p_{2}}-\frac{1}{q_{2}}} \Delta^{m} s_{k}\left(T_{2}\right)\right\|}{\rho_{2}}\right)\right] \\
&<\infty .
\end{aligned}
$$

This implies that $\left\{A k^{\frac{1}{p}-\frac{1}{q}} \Delta^{m} s_{k}\left(T_{1} T_{2}\right)\right\} \in l_{1}$ or $T_{1} T_{2} \in L_{p, q, 1, u, \Delta, A}^{(s)}$. This completes the proof.

\section{Acknowledgment}

This project was funded by the Deanship of Scientific Research (DSR) at King Abdulaziz University, Jeddah, under grant no. (G/586/130/37). The authors, therefore, acknowledge with thanks DSR for technical and financial support.

\section{References}

[1] L. R. Acharya, Linear operators and approximation quantities, Dissertation, I.I.T. Kanpur, India, (2008). 1, 1

[2] A. Alotaibi, K. Raj, S. A. Mohiuddine, Some generalized difference sequence spaces defined by a sequence of moduli in n-normed spaces, J. Funct. Spaces, 2015 (2015), 8 pages. 1

[3] N. De Grande-De Kimpe, Generalized sequence spaces, Bull. Soc. Math. Belg., 23 (1971), 123-166. 1, 1

[4] M. Et, R. Çolak, On some generalized difference sequence spaces, Soochow J. Math., 21 (1995), 377-386. 1

[5] P. Foralewski, H. Hudzik, L. Szymaszkiewicz, On some geometric and topological properties of generalized OrliczLorentz sequence spaces, Math. Nachr., 281 (2008), 181-198. 1

[6] A. Grothendieck, Sur une notion de produit tensoriel topologique d'espaces vectoriels topologiques, et une classe remarquable d'espaces vectoriels liée á cette notion, (French) C. R. Acad. Sci. Paris, 233 (1951), 1556-1558. 1 
[7] M. Gupta, A. Bhar, Generalized Orlicz-Lorentz sequence spaces and corresponding operator ideals, Math. Slovaca, 64 (2014), 1475-1496. 2

[8] P. K. Kamthan, M. Gupta, Sequence spaces and series, Lecture Notes in Pure and Applied Mathematics, Marcel Dekker, Inc., New York, (1981). 1, 1

[9] M. Kato, On Lorentz spaces $l_{p, q}\{E\}$, Hiroshima Math. J., 6 (1976), 73-93. 1

[10] H. Kızmaz, On certain sequence spaces, Canad. Math. Bull., 24 (1981), 169-176. 1

[11] J. Lindenstrauss, L. Tzafriri, Classical Banach spaces, I, Sequence spaces, Ergebnisse der Mathematik und ihrer Grenzgebiete, Springer-Verlag, Berlin-New York, (1977). 1

[12] S. A. Mohiuddine, K. Raj, A. Alotaibi, Generalized spaces of double sequences for Orlicz functions and bounded-regular matrices over n-normed spaces, J. Inequal. Appl., 2014 (2014), 16 pages. 1

[13] J. Musielak, Orlicz spaces and modular spaces, Lecture Notes in Mathematics, Springer-Verlag, Berlin, (1983). 1

[14] J. Patterson, Generalized sequence spaces and matrix transformations, PhD diss., Dissertation, I.I.T. Kanpur, India, (1980). 1, 1.1

[15] A. Pietsch, s-numbers of operators in Banach spaces, Studia Math., 51 (1974), 201-223. 1

[16] A. Pietsch, Eigenvalues and s-numbers, Cambridge Studies in Advanced Mathematics, Cambridge University Press, Cambridge, (1987). 1, 1

[17] K. Raj, S. Pandoh, Some vector-valued statistical convergent sequence spaces, Malaya J. Mat., 3 (2015), 161-167.

[18] K. Raj, S. K. Sharma, Some vector-valued sequence spaces defined by a Musielak-Orlicz function, Rev. Roumaine Math. Pures Appl., 57 (2012), 383-399. 1

[19] W. Rudin, Functional analysis, McGraw-Hill Series in Higher Mathematics, McGraw-Hill Book Co., New YorkDsseldorf-Johannesburg, (1976). 3, 3

[20] E. Savaş, M. Mursaleen, Matrix transformations in some sequence spaces, İstanbul Üniv. Fen Fak. Mat. Derg., 52 (1993), 1-5. 1

[21] Y. Yılmaz, M. K. Özdemir, İ. Solak, M. Candan, Operators on some vector valued Orlicz sequence spaces, F. Ü. Fen ve Mühendislik Bilimleri Dergisi, 17 (2005), 59-71. 1 